\title{
Cosmological Evolution of Supergiant Star-Forming Clouds
}

\author{
Melinda L. Weil ${ }^{1,2}$ \& Ralph E. Pudritz ${ }^{1}$ \\ ${ }^{1}$ Department of Physics and Astronomy, McMaster University, 1280 Main Street W., Hamilton, Ontario, L8S $4 M 1$ Canada \\ ${ }^{2}$ City University of New York, New York
}

9 November 2018

\begin{abstract}
In an exploration of the birthplaces of globular clusters, we present a careful examination of the formation of self-gravitating gas clouds within assembling dark matter haloes in a hierarchical cosmological model. Our high-resolution smoothed particle hydrodynamical simulations are designed to determine whether or not hypothesized supergiant molecular clouds (SGMCs) form and, if they do, to determine their physical properties and mass spectra. It was suggested in earlier work that clouds with a median mass of several $10^{8} M_{\odot}$ are expected to assemble during the formation of a galaxy, and that globular clusters form within these SGMCs. Our simulations show that clouds with the predicted properties are indeed produced as smaller clouds collide and agglomerate within the merging dark matter haloes of our cosmological model. We find that the mass spectrum of these clouds obeys the same power-law form, $d N / d M \propto M^{-1.7 \pm 0.1}$, observed for globular clusters, molecular clouds, and their internal clumps in galaxies, and predicted for the supergiant clouds in which globular clusters may form. We follow the evolution and physical properties of gas clouds within small dark matter haloes up to $z=1$, after which prolific star formation is expected to occur. Finally, we discuss how our results may lead to more physically motivated "rules" for star formation in cosmological simulations of galaxy formation.
\end{abstract}

\section{INTRODUCTION}

The picture of galaxy formation as a hierarchical clustering process (e.g. White \& Rees 1978; Fall \& Efstathiou 1980) has gained considerable observational support over the last decade (e.g. Giavalisco, Steidel, \& Macchetto 1996; Steidel et al. 1998; Abraham et al. 1999). It is thought that evolving dark matter haloes set the stage for the subsequent gas cool' ing and star formation that ultimately result in the plethora of galaxies we see in the present epoch. However, the lack of a detailed physical theory of how stars form in early, evolving environments remains a pressing problem. For example, when gas dynamics and star formation are ignored, distributions of hundreds of dark matter satellites around larger galaxies have been produced in numerical simulations (Klypin et al. 1999; Moore et al. 1999) whereas only tens of satellites are observed. In addition, simulations of galaxy formation in the context of Cold Dark Matter (CDM) dominated universes assume that thermal cooling-function prescriptions can be used to predict how and when gas converts to stars. If it is supposed that the bulk of the cooled gas goes directly into star formation, then the inescapable high cooling rate that occurs in gas within the growing dark matter haloes when the universe was much smaller and denser leads directly to the so-called 'star formation catastrophe'. Far too high a proportion of baryons end up in stars in such simulations compared to the observations. Simulations also repeatedly fail to produce Milky Way types of galaxies because their gas condenses too quickly to be torqued up to the observed angular momentum of gas in spirals (Navarro \& Steinmetz 1997; Weil, Eke, \& Efstathiou 1998; Eke, Efstathiou, \& Wright 2000, hereafter EEW). It is hoped that the remedy for these ills lies in the addition of the feedback of star formation upon the gas. Vigorous supernova activity may heat up the gas enough for it to remain fairly diffuse and extended (e.g. McKee \& Ostriker 1977, McKee 1995).

The purpose of the present paper is to apply the insight of star formation processes in the current epoch to help solve this problem. The observations of star formation in nearby molecular clouds and nearby extragalactic systems clearly show that the prescriptions for star formation imposed in the cosmological models are too simple. Firstly, self-gravitating (molecular) clouds are not supported by thermal pressure, but rather by a combination of turbulent and magnetic pressure (McKee et al. 1993; Pudritz 2000). Thus, simple thermal cooling time arguments are irrelevant for predicting how molecular clouds convert some of their mass into stars. Secondly, stars form in clusters within clumpy subregions of molecular clouds, and the total mass of these embedded clumps is only a few percent of the mass of the entire cloud. Hence, star formation proceeds only in sub regions within molecular clouds that are sufficiently selfgravitating, or equivalently, are at sufficiently high pressure. Thirdly, the mass spectra of molecular clouds and that of their clumps have been found to obey an approximate power law scaling

$d N / d M \propto M^{-1.7 \pm 0.1}$ 
(eg. Blitz 1993, Williams et. al. 2000). The fact that stars form within such special sub-structures in the selfgravitating clouds almost certainly has important implications for the formulation of more physically motivated "rules" for star formation.

Do characteristics of galactic star formation in the present epoch carry over to the epoch of galaxy assembly? Strong indications that the answer is yes come from the old halo globular cluster systems that have been studied around more than 100 galaxies (see review; Harris 2000). Harris and Pudritz (1994, hereafter HP) showed that the mass spectrum characterizing globular cluster systems around a variety of galaxies has nearly the identical form as the mass spectrum for local giant molecular clouds (GMCs) and their clumps. Because the efficiency of conversion of clump gas to stars in bound clusters must be high, the mass spectrum of bound clusters should reflect that of their progenitor clump spectrum. Observations show that globular clusters more massive than $10^{5} M_{\odot}$ and more than a few kpc from the centers of galaxies survive evolution in their host galaxies relatively intact (e.g. Harris, Harris, \& McLaughlin 1999). This is born out by semi-analytical studies of the tidal evaporation and disruption of globular clusters in isothermal dark matter haloes for a very wide range of cluster masses, concentrations, and orbital parameters (e.g. Capriotti \& Hawley 1996). Such calculations show that clusters in the mass range $10^{5}$ to $10^{7} M_{\odot}$ can survive for a Hubble time because they are massive enough to avoid evaporation but not too massive to undergo rapid orbital decay though dynamical friction. Thus, the mass spectrum of the more massive globular clusters around any galaxy essentially preserves the mass spectrum of its progenitor clumps. The numerical model of McLaughlin and Pudritz (1996, hereafter MP) showed that such clump mass spectra arise naturally through an agglomeration/fragmentation process, wherein clumps and clouds build up by the collision and merger of smaller ones within any given dark matter halo, and are destroyed as a consequence of star formation within them.

Globular clusters also provide empirical guidance as to how stars formed during galaxy assembly in a wide variety of systems and environments. The globular cluster luminosity function or, equivalently, mass spectrum, around galaxies has been measured in a variety of galaxy types such as dwarf ellipticals (e.g. Durrell et al. 1996), as well as spirals and ellipticals. The mass spectra are found to be identical to equation (1) within the errors. The mass spectra of globular cluster systems is also independent of the metallicity of the host galaxy (e.g. MP). Moreover, globular clusters are observed to form today in galaxies that have abundant supplies of gas (e.g. Holtzman et al. 1992; Whitmore \& Schweizer 1995; Zepf et al. 1995; Schweizer et al. 1996; Whitmore et al. 1999). Thus, globular cluster formation has a universal character which requires only a sufficient supply of gas, and is independent of host galaxy conditions and details about gas metallicity.

Previous work on this model for cluster formation focussed on processes occurring within some given dark matter halo, and did not fully embed these ideas within a full cosmological framework. Thus, HP and MP constructed a model in which globular clusters form within massive clumps of $10^{5}-10^{6} M_{\odot}$ that are embedded within their yet larger host, supergiant self-gravitating (partially molecu- lar) clouds, denoted SGMCs. The hypothetical SGMC mass range $-10^{7}-10^{9} M_{\odot}-$ is in fact observed for molecular gas within merging or starburst galaxies (e.g. Wilson et al. 2000). In the model, the mass spectrum of the SGMCs (and their substructure - the clumps ) arises from the agglomeration of gas clouds by cloud-cloud collisions within the evolving dark matter haloes. The model envisages gas processes within any given, static dark matter halo but does not include the mergers of such haloes that arise in any hierarchical model for galaxy formation. Thus, globular cluster formation is predicted to occur much as star cluster formation does in molecular clouds at the present day, with the only difference being that huge gas reservoirs were available (through gas infall or ongoing mergers) at these higher redshifts in comparison with current interstellar media.

In the present paper, we undertake, for the first time, a careful examination of the formation of self-gravitating clouds that build up during the assembly dark matter haloes in hierarchical cosmological models. Our purpose is to determine whether or not supergiant clouds form, to measure their mass spectra, and to determine how they evolve from early redshifts to $z=1$. Our high resolution simulations focus, in particular, on the study of these progenitor supergiant clouds before the "star formation catastrophe" occurs (typically at redshifts $z \simeq 1$ ). We specifically designed our simulations to have the resolution needed to study the formation of ensembles of individual, self-gravitating (and partially molecular) clouds that are the actual seats of star formation. The idea is to test and survey the initial conditions for star formation, before problems such as the star formation catastrophe become endemic. This procedure will allow us to test current models of star and globular cluster formation on cosmological scales. It will also ultimately lead to the development of more physically motivated rules for stellar formation in cosmology.

Our results clearly show that SGMCs are commonly formed within evolving dark matter haloes in our first CDM cosmological model, and that they have the the mass spectra and cloud properties predicted for clouds capable of forming globular clusters. We discuss our numerical methods in $\S 2$, and present our results in $\S 3$. Implications of our work for star formation during galaxy assembly and, hence, for globular cluster formation follow in $\S 4$.

\section{NUMERICAL METHODS}

We investigate the formation and evolution of star forming clouds in one of the several viable, spatially flat, CDM cosmological models (Thomas et al. 1998), known as $\tau$ CDM. Structure formation in dark matter and gas is followed using smoothed particle hydrodynamical simulations ( $\mathrm{SPH}$ ). We evaluate the formation of supergiant star-forming clouds during the evolution of small density perturbations initially seeded in an expanding universe.

In order to attain the mass resolution required to identify massive, self-gravitating clouds and their substructure, we choose small dark matter haloes from a large cosmological simulation which has been evolved to the present day $(z=0)$. After tracing the dark matter haloes back to redshift $z=24$, their resolution is improved by increasing the particle numbers by factors of hundreds and adding an equal 
number of gas particles. The volume outside the high resolution region is represented by high-mass, low resolution particles to retain the full gravitational characteristics of the original model. The models are then evolved from $z=24$ to $z=0$. Section 2.1 presents properties of the large cosmological simulation and the method of producing high resolution models. The properties of the dark matter plus gas simulations the code used to evolve the high resolution models are presented in $\S 2.2$.

\subsection{Cosmological Model}

A dark matter only cosmological simulation is used to generate initial conditions for the high-resolution dark matter plus gas simulations described below. The cosmological model is one of two spatially flat CDM simulations by EEW.

The $\tau$ CDM cosmology used in this paper has a matter density parameter $\Omega_{m}=1$, zero cosmological constant, and a Hubble constant $h=0.65$ in units of $100 \mathrm{kms}^{-1} \mathrm{Mpc}^{-1}$. The power spectrum that generates the scale-free initial density fluctuations for these simulations has a shape parameter of $\Gamma=0.2$, which fits observed large-scale correlations in galaxy and cluster surveys (Efstathiou, Bond, \& White 1992). This specific cosmological model could be relevant to the situation in which the late decay of $\tau$ neutrinos produces an additional background of relativistic $e^{-}$and $\mu$ neutrinos. This has the effect of delaying the onset of matter domination. The other large cosmological simulation produced by EEW is a $\Lambda$ CDM model with $\Omega_{m}=0.3$ and $\Omega_{\Lambda}=0.7$. The ages for the $\tau$ CDM and $\Lambda$ CDM cosmological models are 10.0 Gyr and 14.5 Gyr, respectively. Due to its shorter evolution time, we chose to explore the formation of star-forming clouds in the $\tau \mathrm{CDM}$ first. No significant differences in the physical processes related to the evolution of SGMCs are expected for other cosmologies, although the precise time when SGMCs first turn up will of course be different (see $\S 4$ for further discussion). The results on the formation of star-forming clouds in $\Lambda$ CDM models will be reported in a subsequent paper.

The large dark matter simulation represents a cubical volume of the universe of length $L=32.5 h^{-1} \mathrm{Mpc}$ with $N=128^{3}$ dark matter particles. Figure 1 shows a $2-\mathrm{Mpc}$ deep slice of the simulation at $z=0$. EEW identified virialized haloes using a group-finding method, locating over 2000 haloes with particle numbers ranging between about 40 and 30,000. The largest dark matter potential wells are easily seen at the nodal intersections of the filaments. One of the small haloes we chose to resimulate at high resolution is identified by the arrow near the top middle of the figure. This halo contains 65 dark matter particles and has a mass $M=4.5 \times 10^{11} M_{\odot}$.

Haloes to be resimulated at higher resolution are chosen using a set of balanced criteria. Firstly, the mass of each halo is required to be relatively small because computing time increases as the number of particles increases. We represent the high resolution region of the simulation by $N_{d m}=34^{3}$ dark matter particles and $N_{g}=34^{3}$ gas particles. The surrounding low resolution region contains approximately 5000 dark matter particles representing the gravitational forces due the the mass outside the high resolution region. In order to achieve the mass resolution necessary to examine the structure supergiant star-forming clouds, the masses of the small haloes are limited to approximately $5.0 \times 10^{11} M_{\odot}$. Secondly, we choose haloes comprised of enough particles to be physically believable structural entities. The large cosmological simulation is searched for haloes containing $\approx 60$ particles. When expanded to high resolution, the haloes contain gas particles with masses between 2 and $5 \times 10^{6} M_{\odot}$. Thus, while we have sufficient numerical resolution to study the formation of SGMCs, our simulations do not yet allow us to resolve the individual clumps within these clouds that will be the actual sites for the formation of star clusters. In these simulations, the baryon density parameter $\Omega_{b}=0.06$; thus, $94 \%$ of the mass is contained in the dark matter particles. Finally, haloes are required to be well-separated from any other large mass condensations so that computational power will not be exerted upon nearby regions of little interest.

The high resolution initial conditions are created using the method described by Weil et al. (1998). After a halo is chosen at $z=0$, all particles within a spherical volume surrounding it are located at $z=24$, the redshift at which the cosmological simulations commence. A cube just large to contain all the particles is constructed. The length of the cube is typically a few Mpc. Populating the high resolution cube requires displacing particles from a regular lattice in order to represent the spatial fluctuations in density of the cosmology. In order to correctly populate their finer volumes, these high resolution particle displacements require additional calculations that add power above the Nyquist frequency of the original simulation. The higher mass, low resolution region particles are then added in a spherical distribution.

\subsection{Numerical Simulations}

In order to allow statistical analysis of the properties and evolution of star-forming clouds, seven different dark matter haloes were chosen for high resolution resimulation. We studied the evolution of each of these systems, which we designate by distinct Run numbers: $1-7$. Each Run was evolved using the TREESPH code (Hernquist \& Katz 1989). This code treats both dark matter, which is purely gravitational, and gas, which includes hydrodynamical and radiative physics (for details, see Weil et al. 1998).

The gravitational forces between particles are computed using a hierarchical tree algorithm (Barnes \& Hut 1986). Distant particles are grouped into hierarchical cells in which the potential is approximated using a truncated multipole series. The forces on each particle are updated by examining each cell in the tree. The ratio of the size of the cell to its distance from the particle is compared to a defined tolerance parameter to determine whether the cell should be subdivided or the forces due to it calculated as a whole. In this way, computational time is reduced by avoiding explicit two-body force calculations for remote particles, while an accuracy of $\sim 0.1 \%$ is maintained by a tolerance parameter of 0.6 .

The gravitational potential is softened using a cubic spline in order to reduce two-body relaxation. The softening lengths assigned to the particles define the length resolution of the code. For dark matter particles, the softening $\epsilon_{d m}$ is always between 2 and $3 \mathrm{kpc}$. For the gas particles, with masses more than a decade smaller than the dark matter, $\epsilon_{g}$ 


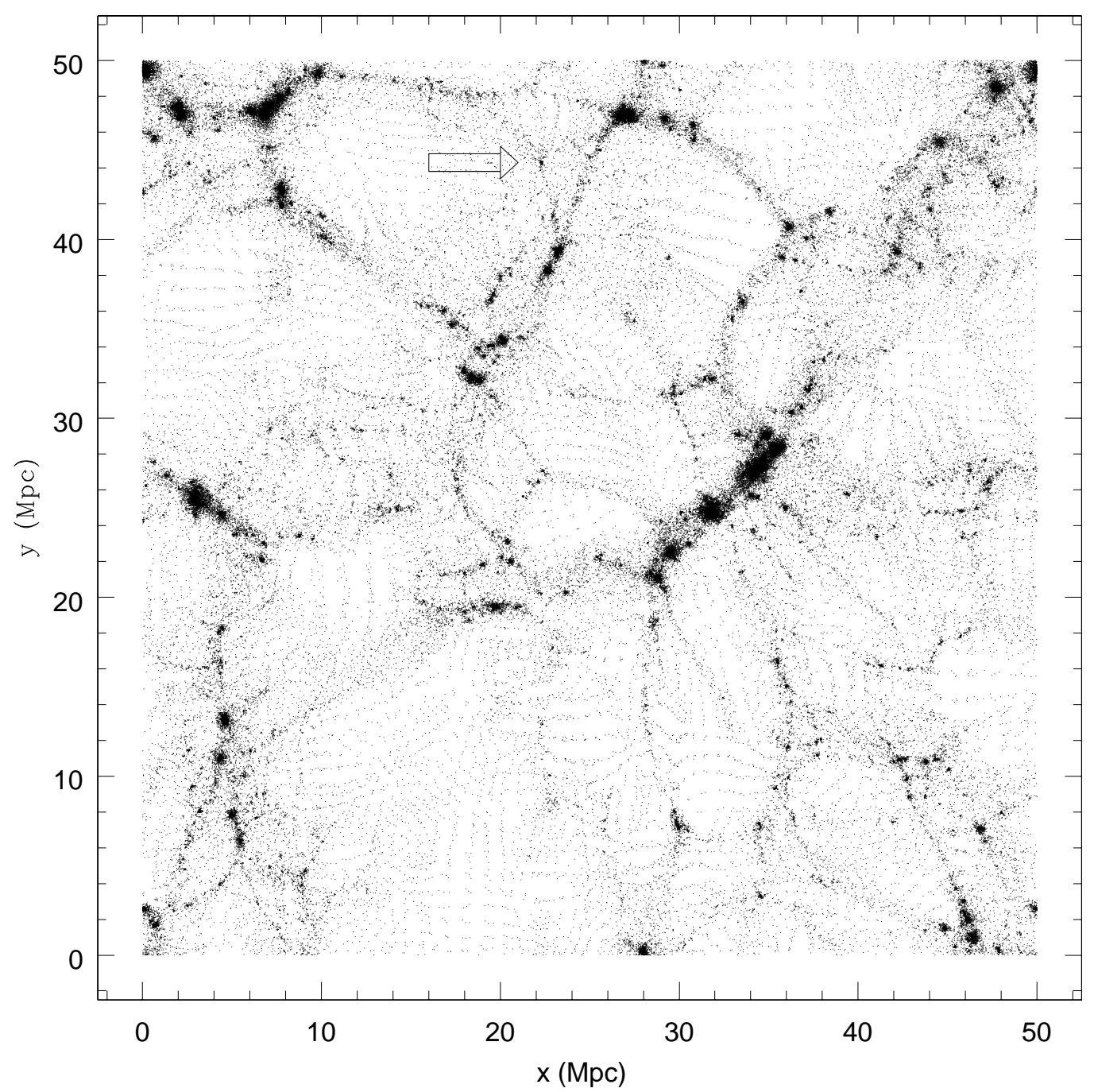

Figure 1. 2-Mpc deep slice of the large, dark matter only cosmological simulation of EEW. The arrow near the top middle points to the small halo which is resimulated at high resolution and shown in Figures 2 and 3.

is between 0.6 and $1.0 \mathrm{kpc}$. This gaseous softening length imposes another restriction on the size of clouds that we can physically resolve, namely, nothing less than several kpc. Given that the HP model suggests a fiducial value of one $\mathrm{kpc}$ for the median SGMC in the distribution, we caution that our simulations will inevitably produce fluffier, lower density clouds by virtue of the limitations imposed by the softening length. Detailed analysis of cloud structure will require future, higher resolution simulations.

Hydrodynamical interactions between gas particles are treated using smoothed particle hydrodynamics (Lucy 1977, Gingold \& Monaghan 1977). The gas is partitioned into self-gravitating fluid elements that evolve according to Lagrangian hydrodynamic conservation laws. Explicitly, pressure gradients and the viscosity of the medium are calculated using conservation of momentum:

$\frac{d \mathbf{r}}{d t}=\mathbf{v}$ and

$\frac{d \mathbf{v}}{d t}=-\frac{1}{\rho_{g a s}} \nabla P+\mathbf{a}^{v i s c}-\nabla \phi(\mathbf{r})$

where $\mathbf{r}$ and $\mathbf{v}$ are vector positions and velocities, $\rho_{\text {gas }}$ is the gas density, $P$ is the pressure, $\mathbf{a}^{\text {visc }}$ comprises the acceleration due to viscosity, and $\phi$ is the gravitational potential. The equation of state if that of an ideal gas; stability during collisional shocks is maintained by an artificial viscosity (Navarro \& Steinmetz 1997).

In order to treat the evolution of the thermal energy, radiative cooling is computed from the ionization, recombination, and cooling rates of Black (1981, Table 3) with modifications by Cen (1992). Analytic approximations to observed rates of collisional ionization, recombination, collisional excitation, bremmstrahlung, and Compton heating and cooling are constructed for H I, H II, He I, He II, and He III. Because we ignore star formation, rather than initializing and evolving metallicities for the gas particles, pri- 
mordial gas is assumed to have a hydrogen mass fraction of $X=0.76$ and a mass helium fraction of $Y=0.24$. As already noted, observations of the IMF and the overall characteristics of the mass functions of globular clusters indicate they are independent of the metallicity of the gas out of which they formed.

The local quantities appearing in the hydrodynamical equations are computed by interpolating between gas particles (Monaghan 1985, Steinmetz \& Müller 1993). Individual smoothing lengths are calculated for each particle in order to ensure that the values for each particle are calculated with reference to 25 to 45 neighbors. In addition to individual smoothing lengths, individual timesteps are permitted for each particle. The largest time step for these TREESPH simulations is $\Delta t=2.5 \times 10^{6}$ years; the smallest number of timesteps in which a particle can reach $z=0$ is $N_{\text {step }}=4000$. Owing to the need to satisfy local stability as required by the Courant condition, particles in the highest density areas are allowed to reduce the size of their timesteps by up to a factor of 32 . In simulations with a large range of mass inhomogeneities, this adaptivity in space and time permits areas of high density to be resolved to an arbitrary resolution.

Our simulations deliberately avoid the implementation of any star formation rule. This enables us to study the properties of ensembles of self-gravitating clouds that build up before any star formation catastrophe - predicted by a naive interpretation of gaseous thermal cooling processes could be important. This assumption implies that we cannot chart the evolution of the metallicity of these early systems before $z=1$. We therefore refrain from speculating about the observed metallicities of globular cluster systems, or any observed trends of this kind (e.g. age-metallicity relations) in any given galaxy, and how these may come to be. Without the high spatial and temporal resolution necessary to resolve and follow the star formation in clumps within SGMCs, such discussion would be premature. The second, and related, limitation is that the destruction of clouds due to star formation is not taken into account. The proper treatment of cloud destruction critically depends however, upon the incorporation of correct star formation physics. Both of these limitations will be relaxed in subsequent work.

The quantities discussed thus far refer to the fiducial initial conditions for our simulations. However, each of the seven haloes was first resimulated at "low resolution." The low resolution simulations were assigned particle numbers for dark matter and for gas of $N_{\text {low }}=22^{3}$ each in comparison to the $N_{\text {high }}=34^{3}$ each of the high resolution simulations. Simulations at this resolution require less than one day to compute on a Sun Ultra 10 workstation. Each halo was evolved from $z=24$ to $z=0$ in low resolution; and was examined for any aberrations that would bias the results. Systems that were in the process of merging or consisted of several subsystems at $z=0$ were excluded. Subsequently, each halo was evolved in high resolution from $z=24$ to $z=1$.

Figures 2 and 3 show the evolution of dark matter and gas, respectively, during Run 2. The length of each panel is $2 \mathrm{Mpc}$ in comoving coordinates and the redshifts are marked at the upper right. In this hierarchical cosmology, several dark matter potential wells, into which star-forming gas falls, have formed by $z=5$. The gas is more centrally concentrated than the dark matter, forming small, denser clumps, surrounded by more diffuse gas. If the first epoch of efficient star formation occurs at $z=5$ in our adopted $\tau$ CDM cosmology, then the present age of the first objects is 9.7 Gyrs. In comparison, if stars first form efficiently at this time in a standard CDM cosmology, their age is $\approx 11$ Gyrs. Even at $z=1$, several gas condensations, which will eventually merge to form a small galaxy, are visible. The present age of stars formed at this time is 6.5 Gyrs in $\tau$ CDM and $\approx 8$ Gyrs in standard CDM. We discuss the significance of these ages in $\S 4$.

\section{RESULTS}

In order to construct a detailed theory for the formation of globular cluster systems (GCS) and, more generally, for star formation within hierarchical cosmologies, the results of simulations need to be compared to observational quantities and models. If stars do indeed form at early epochs in the high pressure clumps within massive gas clouds (as they do in the present epoch), then high resolution cosmological simulations of galaxies should enable the identification of these supergiant clouds. Herein, bound gas masses that represent the star-forming clouds within Runs 1 - 7 are found and examined. The time evolution and statistical and individual properties of these supergiant, star-forming clouds are also investigated.

Our main goal was to identify sufficiently massive bound gas clouds formed in high resolution simulations such as that shown in Figures 2 and 3. Two methods of identifying clumps of particles were applied to outputs at several redshifts of Runs $1-7$. Because the clumps interact with one another and can be irregular, overlapping, and not in dynamical equilibrium, we require an algorithm which can uniquely locate separate clumps and ensure that the systems are bound.

The spherical overdensity algorithm (SO; Lacey \& Cole 1994) grows spheres around density maxima until they reached a defined density contrast with the background. The overdensity $\Delta=178$ corresponds to virialized regions within a uniform collapsing sphere. The friends-of-friends algorithm (FOF; Davis et al. 1985) uses a linking method by which particles are identified as members of a clump if they are closer to the nearest member of the clump than a defined 'link length.' In essence, the link length determines a local density criterium. The results of the two clump-identifying algorithms supports the Lacey and Cole (1994) observation that small clumps are sometimes merged with larger ones for the fiducial parameterizations. The fiducial FOF link length is 0.2 times the mean interparticle separation. Herein, however, the typical link length is defined to be about a factor of two less than that, in order to separately identify small clumps and to avoid including unbound particles. It is likely that, for many clumps, several outlying particles are not included as members; however, the mass contribution of these few particles is negligible in most cases.

For the several output redshifts of Runs 1- 7, all clumps with particle numbers $N_{c, g}>15$ and $N_{c, d m}>30$ for gas and dark matter, respectively, are located. Subsequently, only those clumps whose particles are enclosed within the virial radius of the small galaxy at $\mathrm{z}=0$ are retained. Those clumps 


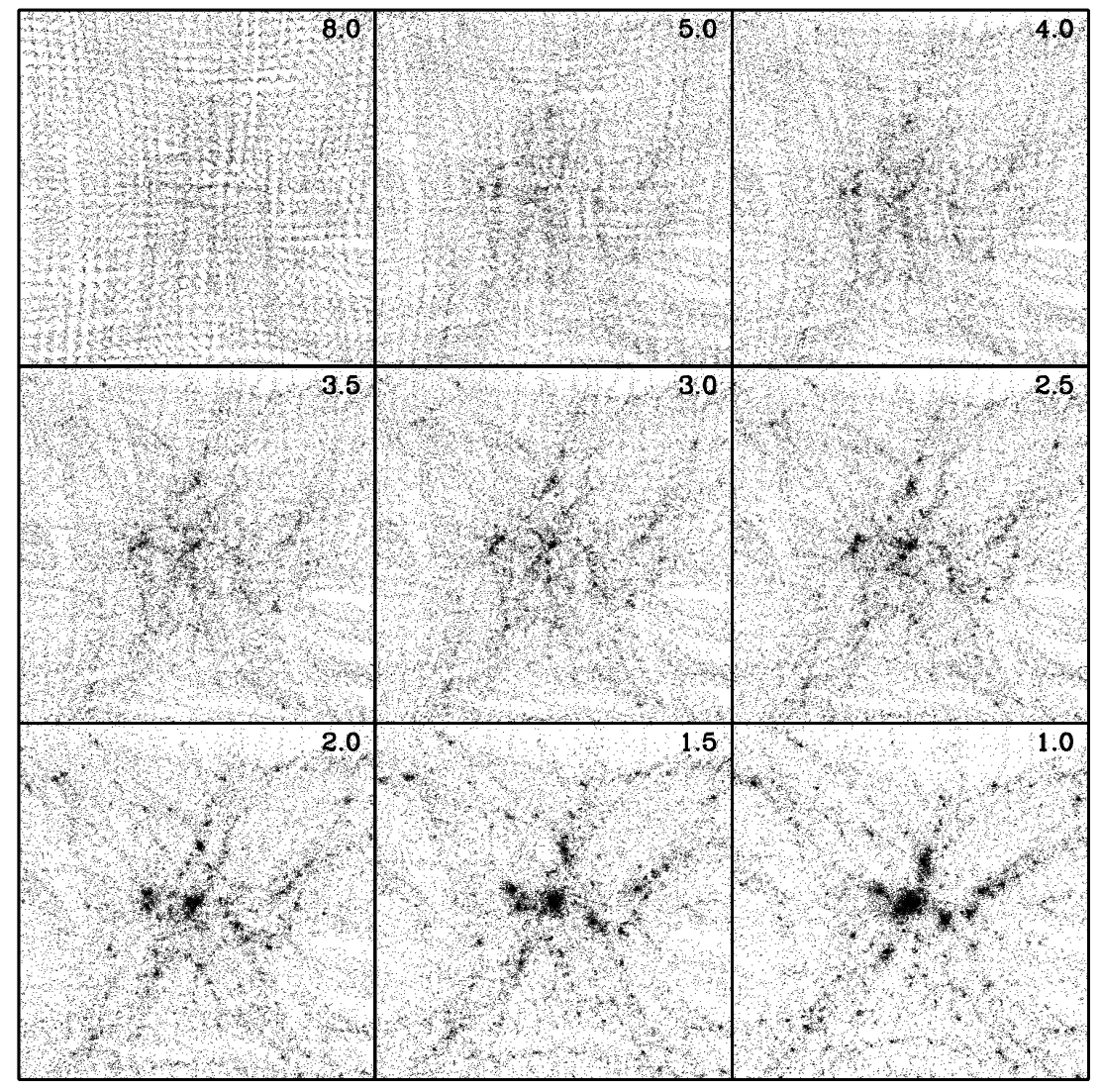

Figure 2. Time evolution of Run 2 dark matter. Each panel measures 2 Mpc in length in comoving coordinates. Redshift is shown in the upper right hand corner of each panel.

with gas masses greater than a few times $10^{7} M_{\odot}$ are considered to be supergiant star-forming clouds. We apply the clump-finding algorithm to three different types of bound objects, namely, to gas plus dark matter agglomerates, to pure gas clouds and, finally, to pure dark matter haloes.

\subsection{Cloud Populations}

The epochs at which star-forming clouds appear are investigated for Runs $1-7$. At our mass resolution limit of a few times $10^{6} M_{\odot}$, by $z=5$ each of Runs $1-7$ produces 2 to 10 gas clouds with masses of several times $10^{7}$ to several times $10^{8} M_{\odot}$. These will eventually become part of the small galaxy. Figure 4 shows the evolution of supergiant starforming clouds located by FOF at eight redshifts for Run 2 . Each of these gas clouds consists of particles identified to be contained within the virial radius of the small galaxy at $z=0$. Each panel measures $1 \mathrm{Mpc}$ in length. In the bottom right panel, the gas particles at $z=0$ are shown. The circle outlines the area within the virial radius of the galaxy, which encloses $4.4 \times 10^{11} M_{\odot}$ (including dark matter and gas). At $z=1$, the clouds contain $1.2 \times 10^{10} M_{\odot}$ gas.

Figure 4 shows the bound gas clouds at eight different redshifts, and their final product at $z=0$. The number of star-forming clouds increases with redshift as the density perturbations of the hierarchical cosmology grow large enough to contain bound material. Although initially wellseparated in space, clouds flow along the filaments visible in Figures 2 and 3 towards the dominant density concentration, piling up in the center as they merge to form a galaxy. The clouds appear irregular in shape throughout all redshifts. It is clear from this figure that clouds are growing by cloudcloud collisions at the same time as the galaxy is building up through a hierarchical merger process.

One can clearly see the collision and agglomeration of clouds in Figure 4. At $z=3.0$, we see 3 distinct clouds in 


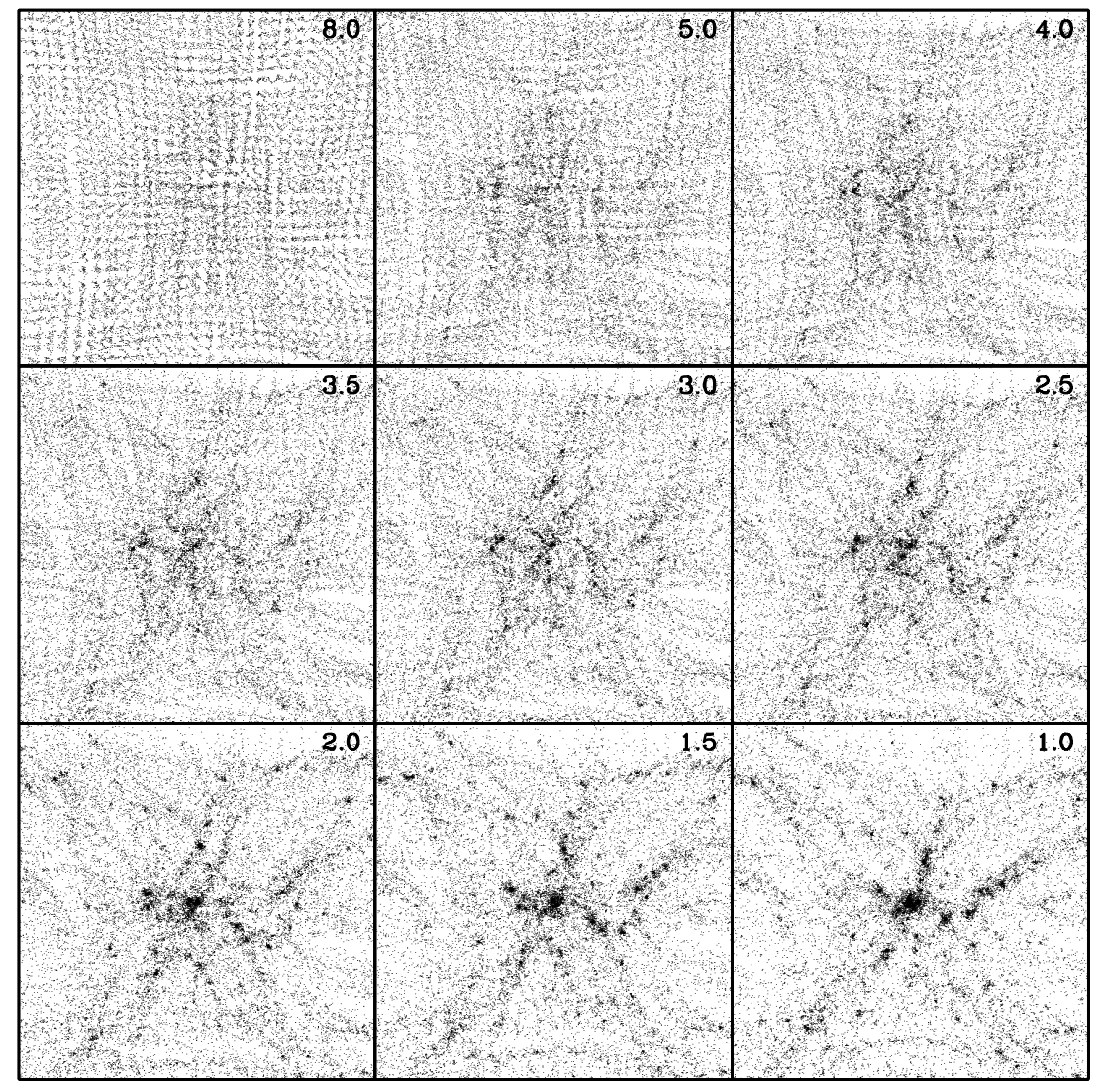

Figure 3. Same as Figure 2 except gas is shown.

middle of the left side that collide, leaving two clouds at that position in the panel at $z=2.5$. As another example, the two amorphous clouds in the upper middle part of the $z=2.5$ panel collide to produce the larger cloud at the same position in the $z=2.0$ panel. Finally, the group of clouds in the middle of the panel at $z=2.5$ collides and agglomerates by $z=1.5$. These, and other details clearly show that gas agglomerates into more massive structures by cloud-cloud collisions. Gas is brought together in two ways: 1) it falls into an individual dark halo and collides with other clouds in that halo, and 2) gas in a given halo collides with the fresh gas contained in other small dark matter haloes that are merging.

Figure 5 shows the central region of Run 2 at $z=1$. The left panel shows the gas and the right panel shows the bound gas clouds. The colors of the clouds are non-physical; all the gas particles that belong to a distinct cloud are represented by a single color. The dark matter particles are not included in this view. As a rule, clouds consist of a dense central core, which contains most of the mass, surrounded by more diffuse gas. We caution that we cannot further resolve the expected substructure within our clouds, because of the limitations imposed by our resolution. In the same way that the halo in the original cosmological simulation of Figure 1 is essentially featureless, these clouds, in which the mass of each gas particle is on the order of the mass of a globular cluster, are not complex. However, if we examine the ensemble of clouds in the center of Figure 5 as a whole, it has the appearance of an amorphous agglomerate containing several high density cores. If the mass resolution for Runs 1 -7 is further increased, the individual clouds would likely show further geometrical complexity.

Each of the seven separate evolving haloes is accompanied by a spectrum of masses for both the gas clouds and the dark matter. Because the number of clouds in each separate Run can be small, the collections of bound objects in each of Runs $1-7$ are added together at each redshift in order to give a larger statistical sampling. At a redshift of 


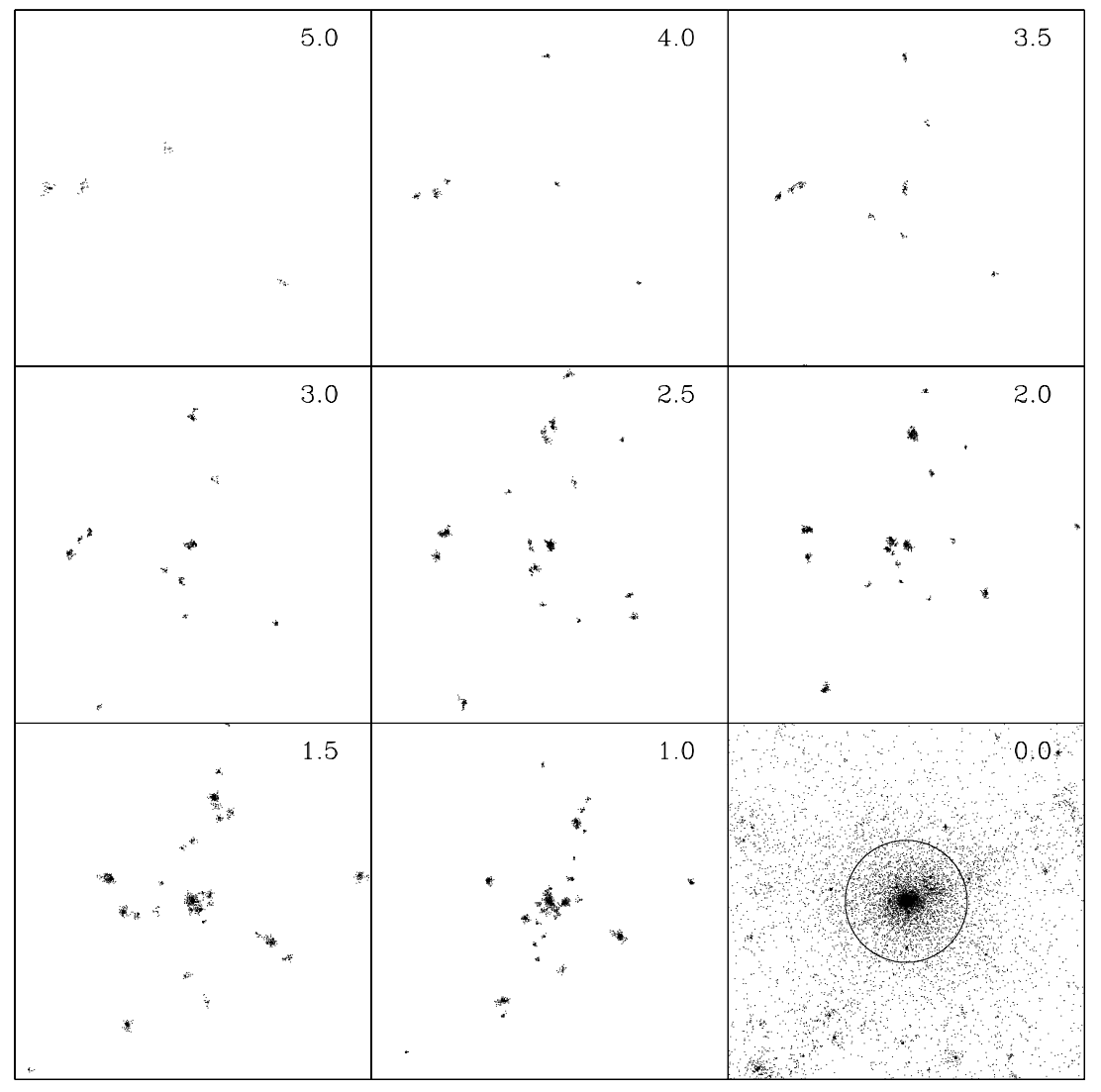

Figure 4. Bound gas clouds at eight redshifts between $z=5$ and $z=1$ for Run 2. The clouds merge eventually to become part of the small galaxy shown at $z=0$. The circle outlines the area within the virial radius. Each panel measures 1 Mpc in length in comoving coordinates.

$z=5$, the haloes consist of between 2 and 11 clouds each. At intermediate redshifts, most haloes consist of about 15 clouds but one contains only 3 and another contains about 30. At $z=1$, half of the haloes contain about 6 clouds because many clouds have merged, one contains about 15, and the rest have about 30 . At $z=5$, the total number of clouds used to calculate the mass spectrum is 30 ; at later redshifts, the total number is greater than 100. Masses are calculated for each cloud. Figure 6 shows mass spectra for all the gas clouds at nine redshifts. Our data appear to flatten slightly at the low mass end, which may be a consequence of our resolution. Remarkably, least squares fits to the logarithmic data show that, at all redshifts, a power law fits the data very well for masses $\geq 10^{7.8} M_{\odot}$, with $1.6 \leq \alpha \leq 1.9$. There is, at best, only very weak evidence for an evolutionary trend for $\alpha$ decreasing over time. The figure indicates that the original low mass clouds accrete mass or merge with other clouds, moving to the right in the panels over time. The low mass clouds are continually replenished as new density condensations grow. The low mass end of the spectrum is not depleted but contains essentially the same number of clouds at all redshifts. Thus, the power law slope is expected to be steeper at high redshift, before high mass clouds have had time to build up, but flattens over time as mass moves to the right in the figure. Because clouds are simply agglomerating without being shredded by feedback from internal star formation (the latter effect was included in MP's model), the upper mass end of the spectrum continues to be pushed to higher masses. It is likely that a correct treatment of star formation within these clouds would result in the truncation of this mass spectrum. At this stage, therefore, the predictions of the analytic HP model and its numerical extension (MP) are seen to arise very naturally within a hierarchical cosmological model. We discuss the ramifications of this in $\S 4$.

Figure 7 shows the mass spectra for the dark matter 


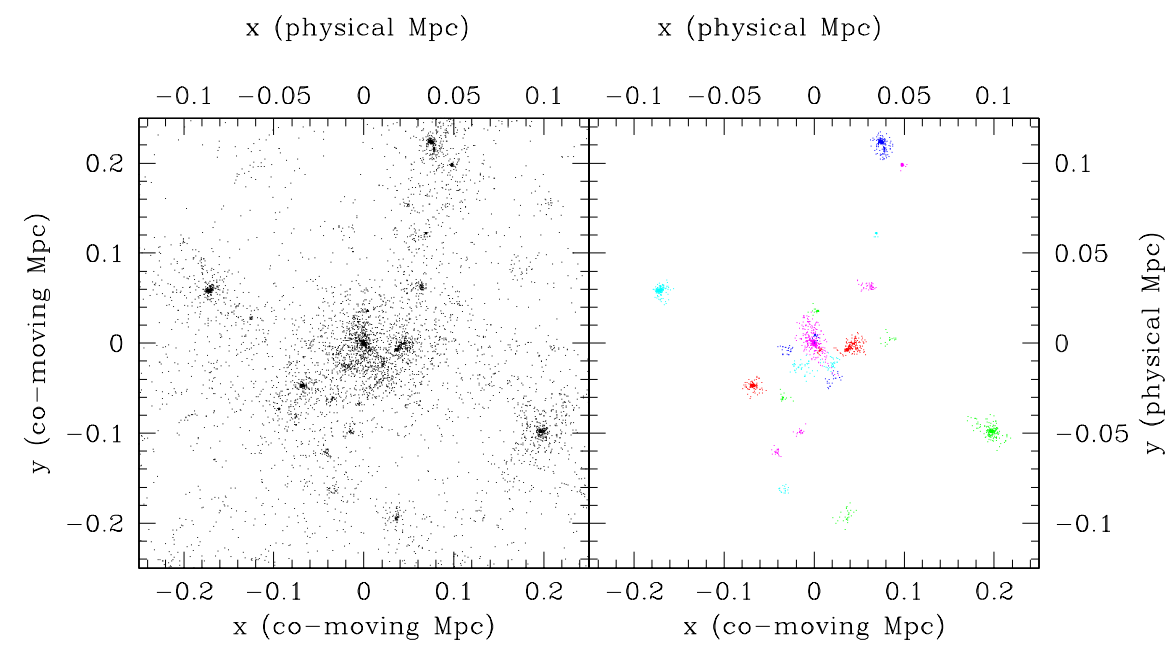

Figure 5. Run 2 at $z=1$. The left panel shows the gas in the central region and the right panel shows the bound gas clouds. Each panel measures $0.5 \mathrm{Mpc}$ in length in comoving coordinates, as labeled on the left and bottom axes. Also shown are physical Mpc on the right and top axes.

in the bound objects. The solid line is the least squares fit. Again, the GCS power law fits well, with $1.5 \leq \alpha \leq 1.8$. The evolutionary trend of the slope is weaker here. If the trend is cosmological, one explanation is suggested by Figures 2 and 3. The dark matter forms potential wells into which gas falls, as seen by comparing the number and density of clumps at any one early redshift, say $z=3$, in the two figures. Once the dark matter clumps are formed, they do not increase in mass as the associated gas does. Figure 7 shows that the mass does not evolve strongly to the right over time, but that most high mass dark matter clumps are in place by $z=3$. The flattening or turnover at small mass is even more apparent for the dark matter than the gas spectra. This turnover also occurs in the globular cluster systems of real galaxies (MP). In observed GCS, it is difficult to determine whether the change in the power law slope is physical or due to incomplete sampling at low masses. In Figure 7, it appears that a collection of small dark matter clumps, with masses less than $10^{9} M_{\odot}$, form at intermediate redshifts but higher resolution simulations are needed to determine if the turnover is physical or due to limited resolution. Meanwhile, we limit the fit to the mass spectra to clumps with masses greater than $3 \times 10^{8} M_{\odot}$.

The dark matter mass spectra in Figure 7 are also compared to the Press-Schechter linear analytic description of the evolution of mass in a hierarchical cosmology (Press \& Schechter 1974). The Press-Schechter multiplicity function predicts how bound objects condense out of a density field described by the cosmological power spectrum $\left|\delta_{k}^{2}\right| \propto k^{n}$,

$N(M, z) d M \propto M^{n / 6-3 / 2} \exp \left[-\left(\frac{M}{M_{*}(z)}\right)^{\frac{n+3}{3}}\right] d M$

where $M_{*}(z) \propto(1+z)^{(-6 /(n+3))}$. The power spectrum of our cosmological simulations is a scale invariant spectrum with $n=1$ but extrapolated to smaller scales (Efstathiou, 


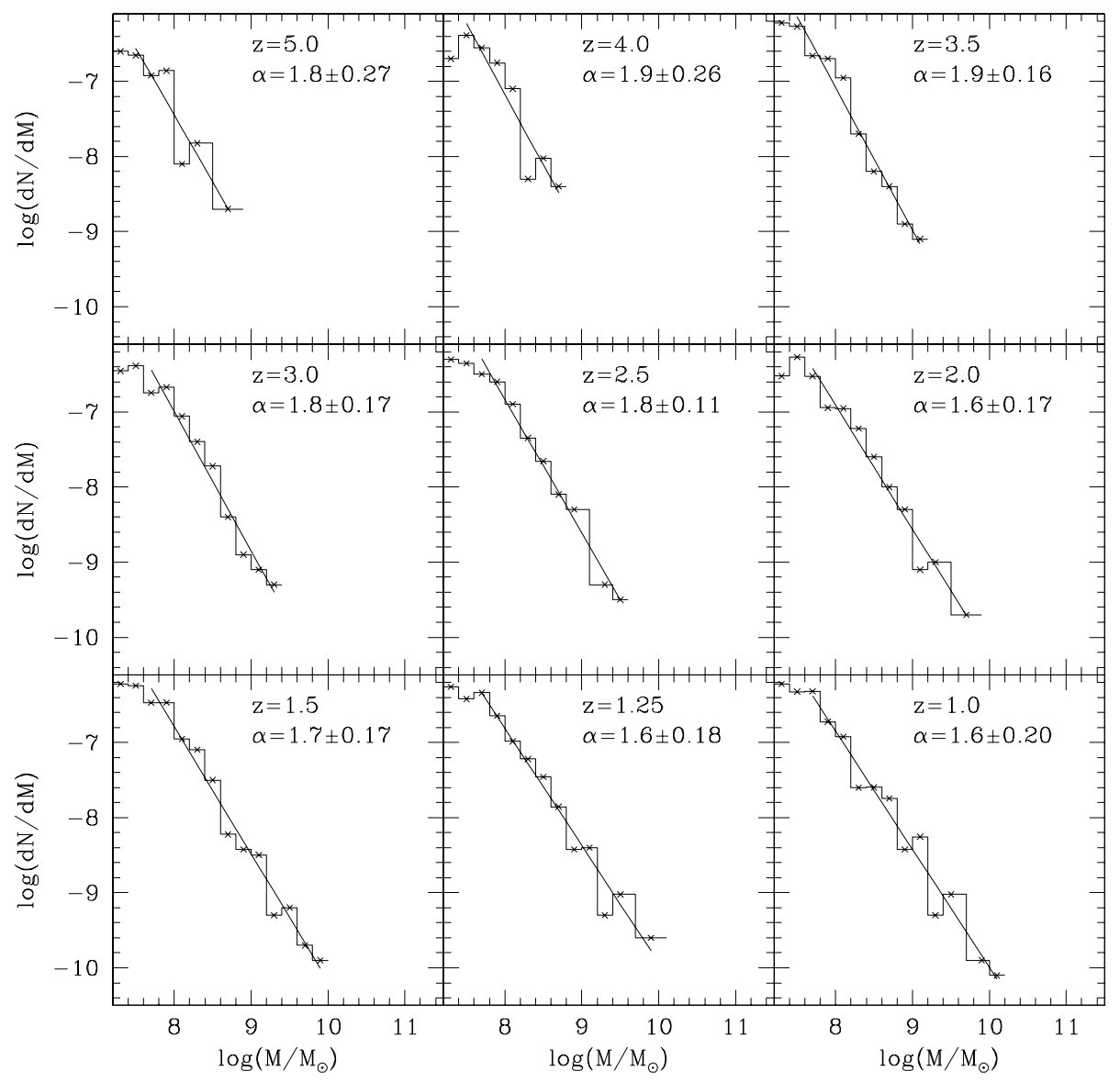

Figure 6. Mass spectrum of combined gas clouds for all seven runs shown at nine redshifts. The solid lines show the power-law fits of eq. (1). The slopes, $\alpha=\Delta \log (d N / d M) / \Delta \log M$, are shown at the top left, below the redshift.

Bond, \& White 1992). Figure 7 shows fits using a non-linear Marquardt method to masses $M>3.5 \times 10^{8} M_{\odot}$. The dotted and dashed lines are for $n=1$ and $n=0$, respectively.

The Press-Schechter method is limited by the assumption of linear growth of bound objects but can be extended to treat non-linear epochs of collapse and merging (e.g. Bower 1991, Bond et al. 1991) Application of the original PressSchechter mass function to our data shows that the fit diverges at high masses where the exponential part of equation (4) is important. In addition, the reliance on redshift in the exponential is weak in the data. Below the exponential cutoff, the fit is a simple power law. However, for $n=1$, the expected slope is -1.3 , flatter than the least squares fit slopes. That agglomeration-produced, power-law models fit the dark matter haloes better than Press-Schechter indicates that the effects of merging overwhelm those of linear growth, as expected in hierarchical cosmologies.

\subsection{Individual Cloud Properties}

Whereas the mass spectrum predicted by observations of globular cluster systems is fit at all redshifts by the supergiant star-forming clouds assumed to be their birthplaces, individual properties of clouds are partially determined by the resolution of the simulations. The HP SGMC properties are based on the application of the virial theorem. They predicted that median SGMC in the power-law spectrum would have a mass $\sim 7 \times 10^{8} M_{\odot}$, a radius $r \sim 900 p c$, surface density $\Sigma \sim 260 M_{\odot} / p c^{2}$, and density $\rho \sim 0.22 M_{\odot} / p c^{3}$. Figure 6 shows that the low mass end fit by the power law is populated by clouds of the expected mass, a few times $10^{8}$ to a few times $10^{9}$.

The assumptions of approximate virial equilibrium for the SGMCs can be tested by noting that the virial theorem for uniform spherical self-gravitating objects predicts that a cloud's mass is related to its radius and internal (1-D) velocity dispersion by $M=5 \sigma^{2} r / G$. The state of our clouds 


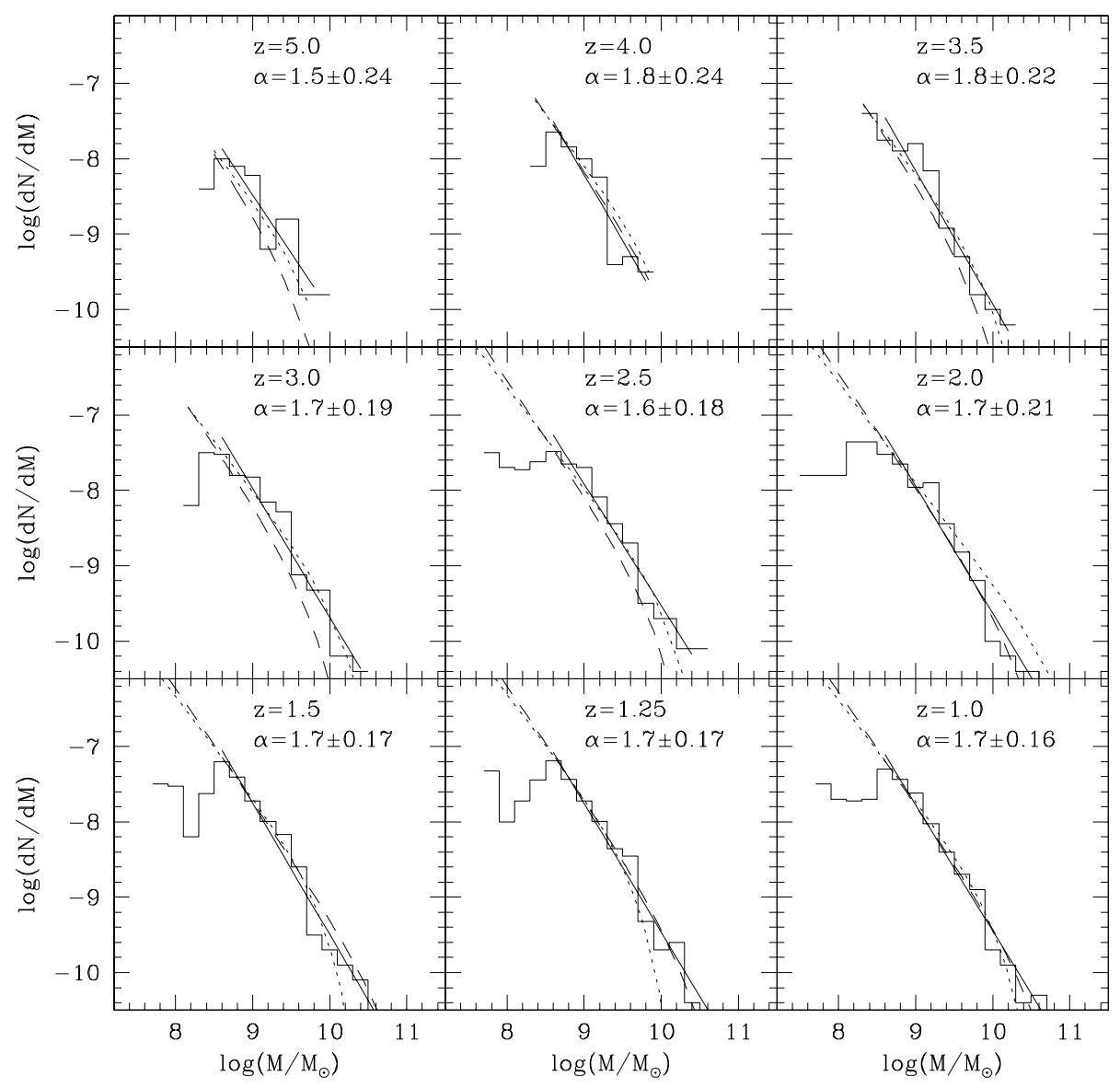

Figure 7. Mass spectrum of combined dark matter clumps for all seven runs shown at nine redshifts. The solid lines show power-law fits, as described in the previous figure caption. The dotted and dashed lines are fits of the Press-Schechter multiplicity function of eq. (1) with $n=1$ and $n=0$, respectively.

is tested by plotting the virial theorem in the form $M$ versus the quantity $\sigma^{2} r$ for the numerical data. Figure 8 shows the logarithmic expression of the quantities for the clouds of Runs $1-7$ at nine redshifts. Least squares fits give slopes, $a$, close to unity, indicating that, as a whole, cloud ensembles are indeed near virial equilibrium. However, Figure 8 also shows that some individual clouds are not virialized but are just condensing out of the primordial density perturbation field or affected by recent interactions (objects of higher than average $\sigma^{2} r$ with masses around $10^{8} M_{\odot}$ as an example).

With gas particle masses of a few times $10^{6} M_{\odot}$, the length resolution in the simulations is limited to $\approx 1 \mathrm{kpc}$. Because the gravitational potential in the simulations is softened in order to avoid two-body effects, the radii of the clouds do not evolve with their mass. Over three decades in mass and between redshifts $z=5$ and $z=1$, cloud radii range from $0.5 \leq r \leq 5 \mathrm{kpc}$. Due to resolution limits, a typical cloud is approximately twice the predicted size. Thus, the typical surface density it a factor of four too low and the typical density a factor of nine too low to be compared directly to SGMCs.

Any model of hierarchical structure formation that contains some prescription for cooling the gas produces an interstellar medium that has both cooler, self-gravitating clouds and a hotter, diffuse medium. The diffuse ISM provides the pressure that truncates the sizes of molecular clouds in our own and other galaxies. Similarly, SGMCs are assumed to be isothermal clouds confined by a surface pressure due to a hotter external medium (e.g. HP).

The temperature structure of the star-forming clouds in Runs $1-7$ cannot explicitly represent that of the interstellar medium because star formation, feedback, and other methods of heating the ISM are not treated in our simulations. Nevertheless, the gas has a temperature structure at $z=1$ in which the temperature of particles in the centers of the clouds is $\leq 10,000 \mathrm{~K}$, the immediately surrounding material 


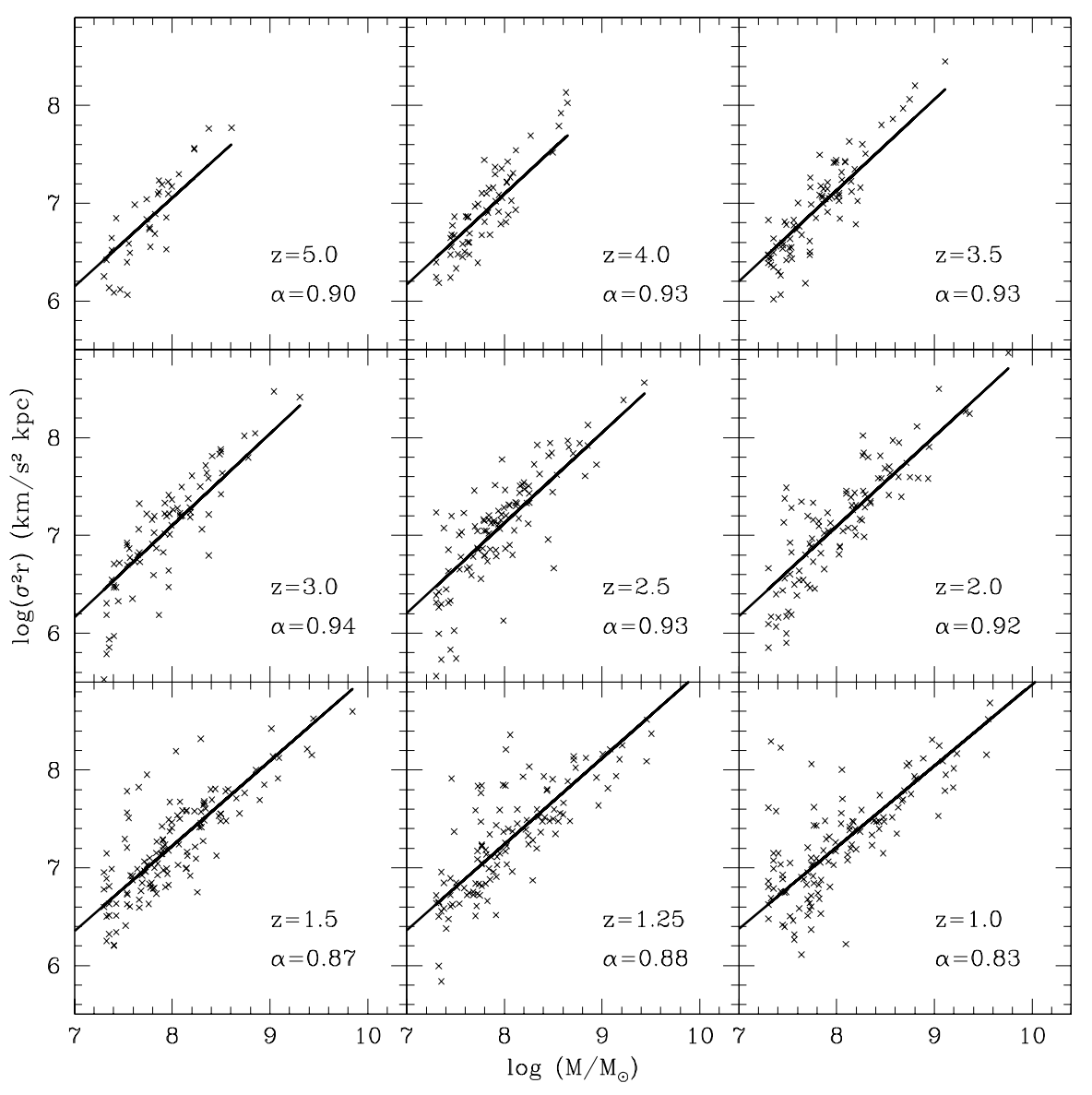

Figure 8. Virial theorem in form $M \propto \sigma^{2} r$ for Runs $1-7$ gas clouds. Redshifts and least squares fits are shown at the top right in each panel.

is between $10,000 \mathrm{~K}$ and $12,000 \mathrm{~K}$, around which particles of $>12,000 \mathrm{~K}$ are smoothly distributed. As expected, the centers of the clouds, where star formation should occur in cool, dense cores, contain low temperature gas, while the more diffuse surrounding medium is hotter. Although not fully representative of the multiphase ISM, the temperature structure is that of a clumpy, warm, neutral medium surrounded by a more diffuse, warm, ionized medium.

\section{DISCUSSION AND SUMMARY}

The simulations clearly establish that, in $\tau$ CDM cosmological models at least, the hierarchical growth of dark matter haloes is accompanied by the growth of self-gravitating, supergiant clouds through the related processes of cloud-cloud collisions and agglomeration. The mass spectrum that we find for SGMCs takes the expected power-law form. That clouds are more concentrated than the dark haloes indi- cates that ISM properties are the key to cloud production. Our SGMCs are not merely condensations in the centers of mini-haloes. Rather, they are self-gravitating entities that are similar to very massive GMCs that form in starburst galaxies today.

We have good indications that these results pertain to other cosmological models as well. We evolved one halo from the $\Lambda$ CDM model mentioned in $\S 2$, and found similar mass spectra and other features of the SGMCs reported here. The generality of our results is, in fact, to be expected on physical grounds because, regardless of the hierarchical model for the dark matter, the gas dynamical processes remain the same. Gas will collapse into the haloes, agglomerate there, and agglomerate with the gas in other nearby haloes that merge with their hosts. Gas agglomeration is a robust process that has been studied for decades in many ISM contexts (e.g. reviews by Elmegreen 1993, Pudritz 2000), beginning with Oort's famous model for gas clouds in the ISM. In a future 
paper, results of a study of gas physics and SGMC formation in $\Lambda$ CDM models will be reported.

It has often been suggested that star formation within assembling galaxies is restricted to the disks of spiral-like galaxies. Thus, it is interesting to observe that the spatial distributions of SGMCs are not disk-like in any of Runs 1-7, but rather are spread out in an irregular fashion throughout the still-assembling dark matter haloes that, at $z=0$, will be the haloes of galaxies with masses of a few $\times 10^{11} \mathrm{M}_{\odot}$. If SGMCs give rise to star and globular clusters, the clusters are likely to be distributed throughout the halo of the galaxy at our epoch.

Several models assume that globular clusters form by fragmenting out of massive, self-gravitating sheets of gas. These sheets could result from cloud-cloud collisions (e.g. Kumai et al. 1993) or swept-up supershells from earlier epochs of primordial star formation (e.g. Brown et al. 1995). Collisions between clouds, it has been suggested, could compress the gas into thin, dense, post-shock sheets that may only be 10 s of parsecs thick. Gravitational fragmentation is then invoked to form clusters by gravitational instability in such self-gravitating layers. Our simulations do not suggest that our SGMCs are significantly crushed by collisions although we hasten to emphasize that our gas smoothing parameter would not allow us to resolve any layer thinner than $0.6 \mathrm{kpc}$. The central point remains: we find that SGMCs are assembled in lower mass potential wells systematically with time, as Figure 6 shows. Cloud-cloud collisions in lower mass dark haloes favors their agglomeration, rather than their disruption, because the cloud-cloud velocity dispersion in lower mass haloes is significantly lower than it is in more massive galaxies that emerge later. Secondly, we note that a major prediction of sheet fragmentation is that there is a preferred mass scale that corresponds to the most rapidly growing, gravitationally unstable mode in the sheet (e.g. Elmegreen \& Elmegreen 1979). The expected, strongly peaked mass spectrum that results from this process is at odds with the observed, power-law mass spectrum of clumps and globular clusters. We suggest that sufficient mass, which can be assembled by a variety of different physical processes - and not external triggers - is all that may be required to form clusters in the ISM of the Milky Way, as well as in assembling galaxies at high redshift (see e.g. HP).

Observations of star formation in current GMCs indicate that the typical star forms as a member of a star cluster. Most star clusters formed in the molecular clouds of our galaxy today become unbound as the natal clumps in their host GMCs are dispersed. The same process may occur for cluster formation in SGMCs. While we anticipate that SGMC internal clumps will be the sites for all of the star formation in the assembling galactic halo, only a few of the putative clusters will be sufficiently bound to survive clump dispersal. Bound clusters become globular clusters. Those that do not remain bound - because of lower star formation efficiency within their natal clumps - will disperse (as the open clusters in our own Milky Way do today) and produce a halo star population. It is likely that these clouds also produce the stars that account for the very faint $\left(28 \mathrm{mag} \operatorname{arcsec}^{-2}\right)$, stellar luminosity observed at large radii around many nearby galaxies (Malin \& Hadley 1999). Proof of these assertions must await further simulations that can include reasonable rules for star formation.
Which aspects of the formation and evolution of starforming clouds are affected by the underlying cosmological model? The most obvious is that the cosmological model dictates the rate at which the dark matter haloes evolve. This, in turn, determines when the gas undergoes substantial cooling and agglomeration. Thus, the model determines the time at which objects such as globular clusters will be produced. The measured ages of the globular clusters can provide an important constraint on any simulation of star formation during galaxy formation. By requiring that cosmological models form SGMCs and, presumably, globular clusters within the age constraints provided by observed globular clusters, the field of viable theories is further narrowed.

Consider the case of our $\tau$ CDM simulations. The earliest time at which SGMCs in the right mass range begin to appear in the simulations is a redshift of order $z \simeq 8$. This is an approximate result; yet higher resolution is needed to determine what occurs at higher redshifts. The oldest that a globular cluster could be in this cosmology is $\sim 10$ Gyr if globular cluster formation began at $z=8$. This is at odds with the latest calculations of globular cluster ages. If the discrepancies that result from distance scale uncertainties are considered, the upper limit of Milky Way globular cluster ages can range anywhere from 12 to 17 Gyr (Grundahl et al. 2000). By invoking further constraints, these authors suggest an age of 16 Gyr for the oldest cluster. Even with this large latitude, $\tau$ CDM models with representative Hubble constants cannot account for globular cluster ages. On the other hand, $\Lambda$ CDM models, with their longer ages due to accelerating expansion, likely provide a reasonably comfortable age to admit globular clusters.

Nevertheless, the lack of feedback from star formation on the process of SGMC assembly is an important limitation. A steep drop in the mass spectrum of globular clusters above several million solar masses is interpreted by $\mathrm{HP}$ as arising from the destruction of SGMCs through supernova activity. A steady-state solution to the agglomeration/fragmentation equations arises if cloud growth is balanced by the destruction of the most massive clouds through disruption by star formation (eg. Oort 1954, Kwan 1979). When the time-scale for forming a yet more massive cloud becomes longer than the cloud's destruction time due to massive stars and supernovae within it, a strong break in the mass spectrum along with a sharp reduction in the number of clouds is seen.

A steady state mass spectrum for the SGMCs does not occur in our models because star formation is ignored. Thus, our SGMCs establish the correct power-law form, and continue to grow to ever larger masses throughout the simulation. The disruption of SGMCs must occur at the very latest by $z=1$, when all indications are that a great deal of star formation must occur. However, globular cluster data suggest that this truncation occurred at higher redshifts when supernovae ripped their natal SGMCs apart (MP). Prescription of a correct rule for star formation will inevitably truncate the mass spectrum of SGMCs.

The fundamental limitation of the simulations presented in this paper is that we are unable to resolve the substructure of the SGMCs. Star formation will take place in the clumps within our clouds, but the expected masses of the clumps is less than the minimum mass of our simu- 
lation particle. As we have made abundantly clear however, it is hazardous to blindly apply star formation rules to the bulk of the gas in an SGMC. An analysis of the onset of star formation must await a future, much higher resolution simulation. Nevertheless, we have clearly shown that we can resolve and study the SGMCs which provide the arenas in which clump, and cluster formation, are likely to take place.

A limitation to all current simulations is that the support of clouds by magnetic pressure, as well as by MHD turbulence of some kind, is not modeled in the purely hydrodynamic codes. This is not as irrelevant as might first appear. Any local molecular cloud undergoes rapid cooling as it forms dues to the efficiency of CO cooling. But, as already noted, this does not lead to catastrophic rates of star formation (i.e. within a dynamical time) throughout the cloud. Catastrophic star formation is prevented because of the effective support provided by MHD turbulence and magnetic pressure within the clouds. Thermal pressure becomes irrelevant. Observations indicate that dynamically significant magnetic fields are present in high redshift galaxies (Kronberg 1994) and, therefore, are an important factor in the physics of any self-gravitating clouds found within them. Magnetic fields could be generated in the turbulent, colliding gas in the assembling dark haloes by localized dynamo processes (e.g. Pudritz \& Silk 1989). Magnetic fields of the required strength (several $\mu$ Gauss) can predate the formation of the first stars and should also be addressed within improved numerical models.

In conclusion, we have found that the supergiant selfgravitating clouds (SGMCs) do indeed form in the dark matter haloes in our $\tau$ CDM simulations. The SGMCs assemble through cloud-cloud collisions as gas collects both by infall into haloes and through the constant merger of gas-containing haloes. A robust power-law mass spectrum for the SGMCs is found in our simulations, wherein, in the mass range from $\approx 10^{7}-10^{10} M_{\odot}, d N / d M \propto M^{1.7 \pm 0.2}$. This is exactly the spectrum that is predicted to account for the formation of globular cluster systems around all types of galaxies (HP). Further independent support for our conclusions comes from studies of chemical enrichment in hierarchical formation of the galactic spheroid, in which an excellent match to the observed metallicity distributions of globular clusters in possible provided that their progenitor clouds have a mass spectrum of the same form as our own SGMC spectrum (Coté et al. 2000).

The properties of the individual SGMCs were also determined in our study. The clouds appear to be in near virial equilibrium. Higher resolution simulations will ultimately provide better determinations of their properties, as well as resolve the substructure of clumps that are expected to be the birthsites of stars and star clusters in the early universe. Finally, preliminary indications are that SGMCs with the same mass spectrum, and formed by similar processes, occur in $\Lambda$ CDM models. These results will be studied and reported in a future paper.

\section{ACKNOWLEDGMENTS}

MLW and REP are especially grateful to Vincent Eke for generously providing initial conditions from his dark matter simulations, without which this project would not have been possible. We thank Bill Harris for interesting discussions and for comments on a draft of our manuscript. REP is indebted to Simon White and the Max-Planck-Institut für Astrophysik for the interest and generous support that he enjoyed during a Research Leave in 1997, when this work was begun. MLW acknowledges the support of a McMaster postdoctoral fellowship from the Dean of Science, Peter Sutherland, and a PSC-CUNY Research Award. The research of REP is supported by grants from the Natural Sciences and Engineering Research Council (NSERC) of Canada.

\section{REFERENCES}

Abraham, R. G., Ellis, R. S., Fabian, A. C., Tanvir, N. R., \& Glazebrook, K. 1999, MNRAS, 303, 641

Barnes, J., \& Hut, P. 1986, Nature, 324, 446

Black, J. H. 1981, MNRAS, 197, 553

Blitz, L., 1993, in Protostars and Planets III, ed. E. H. Levy \& J. H. Lunine (Tucson: Univ. of Arizona Press), 125

Bond, J. R., Cole, S., Efstathiou, G., \& Kaiser, N. 1991, ApJ, 379,440

Bower, R. G. 1991, MNRAS, 248, 332

Brown, J.H., Burkert, A., \& Truran, J.W. 1995, ApJ, 440, 666

Capriotti, E.R., \& Hawley, S.L. 1996, ApJ, 464, 765

Cen, R. 1992, ApJS, 78, 341

Coté, P., Marzke, R.O., West, M.J., \& Minniti, D. 2000, ApJ, 533,869

Davis, M., Efstathiou, G., Frenk, C. S., \& White, S. D. M. 1985, ApJ, 292, 371

Durrell, P. R., Harris, W. E., Geisler, D., \& Pudritz, R. E. 1996 , AJ, 112, 972

Efstathiou, G., Bond, J. R., \& White, S. D. M. 1992, MNRAS, 258, L1

Eke, V., Efstathiou, G., \& Wright, L. 2000, MNRAS, 315, L18 (EEW)

Elmegreen, B. G. 1993, in Protostars and Planets III, ed. E. H. Levy, \& J. I. Lunine (Tucson: Univ. Arizona Press), 97

Elmegreen, B.G., \& Elmegreen, D.M. 1978, ApJ, 220, 1051

Fall, S. M., \& Efstathiou, G. 1980, MNRAS, 193, 189

Giavalisco, M., Steidel, C. C., \& Macchetto, F. D. 1996, ApJ, 470, 189

Gingold, R. A., \& Monaghan, J. J. 1977, MNRAS, 181, 375

Grundahl, F., VandenBerg, D. A., Bell, R. A., Andersen, M. I., \& Stetson, P. B. 2000, ApJ, submitted

Harris, W. E. 2000, in Lectures for 1998 SAAS FEE Advanced Course on Star Clusters (Springer), in press

Harris, W. E., Harris, G.L.H., \& McLaughlin, D.E. 1998, AJ, 115, 1801

Harris, W. E., \& Pudritz, R. E. 1994, ApJ, 429, 177

Hernquist, L., \& Katz, N. 1989, ApJS, 70, 419

Holtzman, J. A., Faber, S. M., Shaya, E. J., Lauer, T. R., Groth, E. J., Hunter, D. A., Baum, W. A., Ewald, S. P., Hester, J. J., Light, R. M., Lynds, C. R., O'Neil, E. J., \& Westphal, J. A. 1992, AJ, 103, 691

Klypin, A., Kravtsov, A. V., Valenzuela, O., \& Prada, F. 1999, ApJ, 422, 82

Kronberg, P. P. 1994, Rep. Prog. Phys., 57, 325

Kumai, Y., Basu, B., \& Fujimoto, M. 1993, ApJ, 404, 144

Kwan, J. 1979, ApJ, 229, 567

Lacey, C., \& Cole, S. 1994, MNRAS, 271, 676

Lucy, L. 1977, AJ, 82, 1013

Malin, D., \& Hadley, B. 1999, in ASP Conf. Ser. 182, Galaxy Dynamics, ed. D. R. Merritt, M. Valluri, \& J. A. Sellwood (San Francisco: ASP), 445

McKee, C. F. 1995, in ASP Conf. Series 80, The Physics of the 
Interstellar Medium, ed. A. Ferrara, C. F. McKee, C. Heiles, \& R. R. Shapiro (San Francisco: ASP), 292

McKee, C. F., Zweibel, E. G., Goodman, A. A., \& Heiles, C. 1993, in Protostars and Planets III, ed. E. H. Levy, \& J. I. Lunine (Tucson: Univ. Arizona Press), 327

McKee, C. F., \& Ostriker, J. P. 1977, ApJ, 218, 148

McLaughlin, D. E., \& Pudritz, R. E. 1996, ApJ, 457, 578

Monaghan, J. J. 1985, Comput. Phys. Rept., 3, 71

Monaghan, J. J., \& Gingold, R. A. 1983, J. Comput. Phys., 52, 374

Moore, B., Ghigna, S., Governato, F., Lake, G., Quinn, T., Stadel, J., \& Tozzi, P. 1999, ApJL, 524, 19

Navarro, J. F., \& Steinmetz, M. 1997, ApJ, 478, 13

Oort, J. H. 1954, Bull. Astron. Inst. Netherlands, 12, 177

Press, W. H., \& Schechter, P. 1974, ApJ, 187, 425

Pudritz, R. E. 2000, in ASP Conference Series, From Darkness to Light, ed. T. Montmerle \& P. André (San Francisco: A.S.P.), in press

Pudritz, R. E., \& Silk, J. 1989, ApJ, 342, 650

Schweizer, F., Miller, B. W., Whitmore, B. C., \& Fall, S. M. 1996, AJ, 112, 1839

Steidel, C. C., Adelberger, K. L., Dickinson, M., Giavalisco, M., Pettini, M., \& Kellogg, M. 1998, ApJ, 492, 428

Steinmetz, M., \& Müller, F. 1993, A\&A, 268, 391

Thomas, P.A. et al. 1998, MNRAS, 296, 1061

Weil, M. L., Eke, V. R., \& Efstathiou, G. 1998, MNRAS, 300, 773

White, S. D. M, \& Rees, M. J. 1978, MNRAS, 183, 341

Whitmore, B. C., \& Schweizer, F. 1995, AJ, 109, 960

Whitmore, B. C., Schweizer, F., Leitherer, C., Borne, K., \& Robert, C. 1993, AJ, 106, 1354

Whitmore, B. C., Zhang, Q., Leitherer, C., Fall, S. M., Schweizer, F., \& Miller, B. W. 1999, AJ, 118, 1551

Williams, J. P., Blitz, L., \& McKee, C. F. 2000, in Protostars and Planets IV, ed. A. P. Boss, S. S. Russell, \& V. Mannings (Tucson: Univ. of Arizona Press), 97

Zepf, S. E., Carter, D., Sharples, R. M., \& Ashman, K. M. 1995, ApJL, 445, 23 